\title{
Evaluación de la competencia profesional de residentes de Medicina General Integral para la atención a la familia
}

\author{
M. Rocha-Vázquez, M.A. San Juan-Bosch
}

Introducción. El proceso de evaluación de las competencias para diagnóstico de necesidades de aprendizaje es un elemento de vital importancia para el perfeccionamiento continuo del personal de salud; por ello, el presente trabajo pretendió evaluar la competencia de los residentes de Medicina General Integral en la atención a la familia, área de acción de estos profesionales de la salud. Sujetos y métodos. Para lograr el objetivo propuesto se ejecutó una investigación de evaluación, con diseño cuanticualitativo, en la Facultad de Ciencias Médicas de Cienfuegos, desde noviembre de 2005 a enero de 2007. De un total de 64 residentes de segundo año se seleccionaron 25 de manera aleatoria, que representan el 39\% del total, a los cuales se les aplicó un examen de competencia, y cada uno de ellos efectuó dos visitas a la familia. Resultados. Sólo el 11,8\% de los residentes aprobó el examen aplicado; las mayores dificultades se centraron en los temas de intervención familiar, evaluación del funcionamiento familiar y determinación de la salud familiar. De igual forma se constataron deficiencias en la ejecución de las visitas a la familia en cuanto a cumplimiento de sus principios, violación de etapas e intervenciones necesarias, fundamentalmente. Conclusiones. Con los instrumentos aplicados y los criterios asumidos para esta investigación se observa un muy bajo nivel de competencia de los residentes para la atención a la familia, lo que alerta de que deben diseñarse e implementarse estrategias inmediatas para revertir esta situación.

Palabras clave. Atención familiar. Competencia profesional. Residentes.

Evaluation of the professional competition of residents of Integral General Medicine for the attention to the family

Introduction. The process of evaluation of the competitions for diagnostic of learning necessities is an element of vital importance for the personnel's of health continuous improvement, it is for it that the present work sought to evaluate the competition of the residents of Integral General Medicine in the attention to the family, area of these professionals' of the health action. Subjects and methods. To achieve the proposed objective an evaluation investigation it was executed, with quanti-qualitative design, in the Medical School of Medicine of Cienfuegos, in the period of November 2005 to January of the 2007. Of a total of 64 second year-old residents they were selected in a random way 25 that represent 39\% of the total, to which were applied a competition exam and two visits were observed to the family executed by each one of them. Results. Alone $11.8 \%$ of the residents approved the applied exam, being centered the biggest difficulties in the topics of family intervention, evaluation of the family operation and determination of the family health. Of equal he/she is formed they verified deficiencies in the execution from the visits to the family as for execution of their principles, violation of stages and necessary interventions fundamentally. Conclusions. With the applied instruments and the approaches assumed for this investigation, very low level of the residents' competition it is translated for the attention to the family, what alerts that we should design and to implement immediate strategies to revert this situation.

Key words. Family attention. Professional competition. Residents.

\section{Introducción}

La calidad de un servicio de salud parte del nivel de competencia y desempeño de sus trabajadores en el cumplimiento de sus funciones laborales y sociales. Por lo tanto, el análisis del factor 'recurso humano' es clave y dentro de él, básicamente, nos estamos refiriendo a lo que 'sabe y
Facultad de Ciencias Médicas Doctor Raúl Dorticós Torrado. Cienfuegos, Cuba.

Correspondencia Dra. Mabel Rocha Vázquez. Facultad de Ciencias Médicas Doctor Raúl Dorticós Torrado. Edif. 18 plantas, n. ${ }^{\circ} 19$, piso 16 , apartamento 1601. Pastorita. CP 55100 Cienfuegos, Cuba.

E-mail mrv@ucm.cfg.sld.cu 
sabe hacer' (competencia) y a lo que en realidad 'hace' (desempeño) [1].

Desde sus comienzos, la medicina familiar ha promovido la idea de que un médico de familia debe relacionarse con el individuo, la familia y el contexto social; un componente fundamental de esta especialidad es la comprensión de los problemas de la familia y su solución [2,3].

El diseño del sistema de formación del especialista en el subsistema de la educación de posgrado en Cuba tiene como objetivo fundamental la superación constante y sistemática del personal de salud. En Cuba, la especialidad de medicina familiar se denomina Medicina General Integral (MGI); es un proceso de formación académica de posgrado a partir de un médico general integral básico, que requiere seis años de formación de pregrado, con un perfil profesional orientado hacia la atención primaria de salud.

\section{Breve reseña del programa de formación de residentes en MGI vigente en Cuba}

El actual programa de residencia de MGI consta de dos años de duración. Cada año académico se planifica para 48 semanas, de ellas 45 se dedican a desarrollar el programa docente y tres a la preparación y realización de los exámenes de promoción. Tiene una estructura modular, con un total de 29 módulos, de los cuales 25 se desarrollan en la atención primaria de salud y cuatro en los hospitales, e incluye además tres cursos, tres rotaciones y una estancia en Medicina Natural y Tradicional [4]. Incluye el despliegue de objetivos y contenidos de cada módulo; dentro de los correspondientes al primer año se encuentra el módulo de Salud Familiar, que se desarrolla durante cuatro semanas.

\section{Breve reseña sobre el módulo de Salud Familiar}

El objetivo del módulo es identificar y evaluar los problemas de salud que afectan a la familia y realizar acciones integrales para su solución.

Dentro de sus contenidos se enuncian: la familia como sistema, criterios para evaluar la salud familiar, clasificación de la familia según la salud familiar, tipos de intervención familiar, planificación familiar, embarazo, aborto, crecimiento y desarrollo, y adolescencia.

En el programa de residencia de MGI y en el manual de procedimientos del médico y la enfermera de la familia en Cuba se define la atención médica integral $[5,6]$, que llevada al contexto familiar se traduce en la atención médica integral a la familia [7].

Constituye una necesidad que el médico en la atención primaria de salud posea una sólida preparación que le permita 'saber' y 'saber hacer' para conducir exitosamente el proceso de atención a la familia [7-10]. La sociedad exige del personal de salud que se establezcan normas particulares de ejercicio profesional [11], pero aún se identifican en la familia cubana necesidades de atención. Por ello que decidimos evaluar la competencia profesional de los residentes de MGI en la atención a la familia.

\section{Sujetos y métodos}

La presente investigación es una evaluación, con diseño cuanticualitativo, realizada en la Facultad de Ciencias Médicas de Cienfuegos, en el período de noviembre de 2005 a enero del 2007, para analizar las competencias profesionales de residentes de MGI para la atención integral a la familia. El emplazamiento fue el municipio de Cienfuegos y como muestra se conformaron dos grupos:

- Grupo I. Formado por los 25 residentes de segundo año de MGI, que representa el universo de los residentes del municipio de Cienfuegos. Se seleccionaron los residentes de segundo año por encontrarse en año terminal de la residencia, tras haber seguido el módulo de Salud Familiar y hallarse en etapa de exámenes estatales de la especialidad, próximos a graduarse como especialistas. Ello permite realizar valoraciones acerca de su nivel de competencia, ya que teóricamente, y en el orden práctico, su formación ha concluido y sólo queda pendiente la realización del examen estatal.

- Grupo II. Formado por un total de 50 familias atendidas por los residentes incluidos en el estudio y seleccionadas al azar simple (dos por cada residente). Como parte de nuestro programa de medicina familiar, los profesionales médicos de la atención primaria de salud visitan al $100 \%$ de las familias anualmente para evaluarlas; para ello realizan una programación. Entonces, la autora principal del trabajo acompañó a los residentes a dos de las visitas que tenían programadas, con el fin de realizar la observación de las 
actividades y la recogida de información. según la matriz de evaluación diseñada al efecto.

Se realizó un examen de competencia a la totalidad de los residentes incluidos en el estudio y la observación de visitas a la familia ejecutadas por estos residentes. Para el examen de competencia se confeccionó un examen tipo test objetivo, que de forma sintética es aquel en el cual el sujeto elige su respuesta dentro de varias respuestas proporcionadas por el elaborador del examen, como ítems de respuesta alternativa (verdadero o falso), ítems de selección múltiple, ítems de igualamiento o pareación...

El examen incluyó elementos relativos a aspectos conceptuales, representación gráfica de la familia, evaluación del funcionamiento familiar, intervención familiar y determinación de la salud familiar.

La clave de calificación se elaboró para cada pregunta y se definió, de igual forma, el patrón de aprobado y no aprobado. En este caso, definimos obtener el 70\% de los puntos para estar aprobado, considerando un total de 100 puntos en el examen. De esta forma cumplimos con los elementos del diseño de un examen según Salas-Mainegra [12].

Para la observación de visitas a familias realizadas por los residentes se elaboró una guía de observación de la actividad por parte de la autora.

Para registrar la información se utilizaron las siguientes variables: objetivos de la entrevista, cumplimiento de sus principios y etapas, observación y registro de la misma, criterios sobre necesidad de intervención familiar y tipo (de ser necesaria), intervención realizada y nivel de desarrollo y participación en la asistencia familiar ejecutada, así como la correspondencia entre la intervención requerida y la realizada. Los criterios para la operatividad de las variables fueron los abordados por Louro-Bernal et al [13] al describir los elementos de la entrevista familiar.

El examen de competencia y la guía de observación de la visita a la familia fueron sometidos al criterio de expertos. Para seleccionar a los expertos se tuvo en cuenta la definición de OñateRamos [14]: 'se entenderá por experto tanto al individuo en sí como a un grupo de personas $\mathrm{u}$ organizaciones capaces de ofrecer valoraciones conclusivas de un problema en cuestión y hacer recomendaciones respecto a sus momentos fundamentales con un máximo de competencia'.

Las características esenciales de los expertos están dadas por: competencia, creatividad, dis- posición a participar, capacidad de análisis y de pensamiento, y espíritu colectivo y autocrítico.

Sobre la base de estos criterios, nuestro grupo de expertos estuvo integrado por:

- Profesores con más de 10 años de experiencia en la atención primaria de salud y, en particular, en el trabajo con familias.

- Profesores del Departamento de MGI.

- Profesores del Departamento Docente Metodológico de la Facultad de Ciencias Médicas.

Se valoró el nivel de competencia de los expertos mediante la determinación del coeficiente de competencia o coeficiente $\mathrm{K}$, según metodología de Oñate-Ramos [14].

El número total de expertos seleccionados fue de 10; de ellos, nueve obtuvieron un coeficiente de competencia alto según la metodología utilizada y uno obtuvo un coeficiente medio.

Las preguntas formuladas a los expertos estuvieron dirigidas a explorar los criterios siguientes: fiabilidad (grado de correspondencia entre las preguntas del instrumento evaluador y los contenidos a evaluar), validez (precisión y estabilidad de la medición que se realiza) y capacidad de generalización (si no se detectaban diferencias entre los territorios, ocho áreas de salud del municipio, se concluiría que los resultados del instrumento evaluador aplicado eran generalizables) de los instrumentos aplicados. Los 10 expertos coincidieron en que el instrumento empleado resultó fiable, válido y generalizable.

\section{Resultados}

\section{Resultados del examen de competencia aplicado a residentes}

\section{Criterios sobre el instrumento}

Para analizar el criterio de los residentes sobre el examen aplicado se consideraron cinco categorías, desde 'muy difícil' hasta 'muy fácil'. Como aspecto relevante podemos plantear que el $66,7 \%$ de los médicos evaluados encontraron 'difícil' el instrumento aplicado. Se analizó también la repercusión del examen aplicado en los conocimientos de los residentes, como resultado de lo cual se obtuvo que del total de médicos encuestados, la mayoría consideró que consolidó conocimientos $(61,1 \%)$. 


\section{Resultados obtenidos en el examen}

Del total de residentes examinados, sólo el 11,8 \% aprobó el examen. La media del grupo de estudio para la nota final fue de 45,2 puntos sobre los 100 posibles.

\section{Resultados de la calificación por preguntas}

Para este análisis tuvimos en cuenta el porcentaje de aprobados por preguntas en los diferentes temas. Resultó evidente que la temática de representación gráfica de la familia fue la de mejores calificaciones, con un 77,7 \% de aprobados, seguido de los aspectos conceptuales, con un 55,5\%. Si reflexionamos además sobre el nivel de asimilación que miden las preguntas, nos percatamos de que preguntas como la que evalúa la determinación de la salud familiar no supera el 50\% de aprobados. Se utilizaron preguntas que simulan situaciones reales que se presentan en la práctica cotidiana, donde el residente debía aplicar los conocimientos previos; por ejemplo, en la evaluación del funcionamiento familiar, habilidad ésta que debe sistematizarse, sólo obtuvimos un 5,9\% de aprobados.

\section{Resultado de la observación de visitas a familias}

Lo primero que evaluamos en la observación de la visita fueron los objetivos propuestos. En la mayoría de los casos fue para obtener información sobre la caracterización y evaluación familiar (47,0\%); preocupa que el 17,6\% acudió sin objetivos previos, lo cual evidencia la falta de planificación de la actividad. La improvisación puede llevarnos a cometer errores y, siempre que sea posible, la visita a la familia debe pensarse con anterioridad.

\section{Cumplimiento de los principios de la entrevista} El principio que casi siempre se cumple es la utilización de un lenguaje claro $(94,1 \%)$ y, en menor medida, la neutralidad del investigador (76,5\%). Sin embargo, no podemos decir lo mismo del carácter grupal, que se logró en el 47,0\%, y sólo el $5,9 \%$ de los residentes cumplieron el carácter circular en las visitas realizadas.

\section{Desarrollo de las etapas de la entrevista}

- Etapa de preparación previa. Determinaron objetivos y roles el 70,6\%, determinaron lugar el $88,2 \%$, y sólo el $47,0 \%$ citó a la familia previamente.
- Etapa social. La totalidad de los residentes cumplimentó el recibimiento, saludo y acomodo, y la mayoría, la presentación (94,1\%). El 76,4\% formuló preguntas relacionadas con el entorno de vida material. Mayores dificultades presentó el encuadre de tiempo, pues sólo el $29,4 \%$ lo hizo.

- Etapa de interacción. En la totalidad de las visitas se formularon las preguntas de forma adecuada; en el $82,3 \%$ de los casos el entrevistador brindó confianza a la familia, pero sólo en el 35,2\% de las familias los entrevistados expresaron percepción respecto a los problemas de salud de la familia y la forma de contribuir a su solución, lo cual evidencia una actitud pasiva a pesar de haberse estimulado en la mayoría de las visitas la interacción comunicativa $(64,7 \%)$.

- Etapa de cierre y compromiso. En el 76,5\% de las visitas se realizó un resumen destacando las potencialidades de la familia, se establecieron compromisos en el 70,6\% de casos, y en mucha menor medida se puntualizaron los problemas detectados y se redujeron tensiones, con un 52,9 y $47,0 \%$, respectivamente.

\section{Observación de la entrevista}

La mayoría de los residentes se mantuvieron atentos a todos los aspectos que deben observarse en la entrevista $(88,2 \%)$.

\section{Registro de la entrevista}

Se logró por parte del 76,5\% de los residentes.

Familias tributarias de intervención familiar Según criterio del observador, el 58,8\% de las familias visitadas eran tributarias de intervención y cuando contrastamos con las realizadas, sólo se ejecutó el $70 \%$ de ellas.

\section{Nivel de desarrollo y participación familiar en la asistencia familiar ejecutada} El $100 \%$ de los casos se encuentra en el nivel II de los descritos por Doherty-Baird [12], es decir, recibe y aporta información y da consejo médico a la familia.

\section{Tipos de intervención familiar requeridas}

La totalidad de las familias tributarias de intervención, según criterio del observador, requerían orientación familiar y la totalidad de las intervenciones realizadas se correspondieron con ésta. 


\section{Discusión}

Al existir un porcentaje elevado de residentes que valora el examen aplicado entre 'difícil' y 'muy difícil' -reconociendo que con el solo hecho de responder el examen han consolidado y aprendido contenidos-, se evidencia que, con relación al tema 'familia', quedan elementos a perfeccionar en la formación de nuestros residentes en un área del saber que es propia de la especialidad y que nos distingue de otros profesionales.

El bajo porcentaje de aprobados en el examen aplicado constituye una alerta sobre el nivel de competencia en este tema y sobre la necesidad de actuar inmediatamente para revertir esta situación.

Estudios realizados en Cuba por Louro-Bernal [3] y Bedoya [15] encontraron insuficiencia en los conocimientos y capacidades para realizar evaluaciones e intervenciones familiares; de igual forma, una investigación realizada por CabreraPivaral et al [16] sobre aptitud clínica del médico familiar evidenció limitaciones para identificar y orientar la disfunción familiar.

Un estudio realizado en México [17] obtuvo resultados similares a los nuestros, donde el $82 \%$ de los residentes se ubicó con niveles bajos y muy bajos en la aptitud clínica de manejo familiar. Estos resultados pueden denotar escasa reflexión en el manejo de las familias, así como la existencia de ambientes académicos poco propicios, variables susceptibles de indagación en posteriores investigaciones.

Díaz et al [18] plantean que, a consecuencia de la elevada presión asistencial que padecen los médicos, la mayor parte del tiempo lo destinan a consultas a demanda, en detrimento de la actividad programada, de la atención domiciliaria y de las actividades no asistenciales.

Con relación a la etapa de interacción, compartimos el criterio de otros investigadores según los cuales 'los lazos de confianza de la relación médico familiar/persona-familia son inherentes al ejercicio de la medicina familiar. Constituyen la base de una relación profesional ganancial bilateral, valora a los individuos en todas sus dimensiones y considera lo importante que es la familia en el continuo salud/enfermedad' [19].

Los resultados obtenidos denotan un bajo nivel de competencia de los residentes para la atención a la familia, con los instrumentos aplicados y los criterios asumidos para esta investigación, lo que implica que debemos diseñar e implementar estrategias para revertir esta situación, teniendo en cuenta que la medicina familiar constituye la práctica hegemónica en Cuba y donde la atención familiar pasó de ser una área indiferenciada a un campo de actuación propio, con enfoque y paradigma definido y contenido claro, con lo cual debemos ser consecuentes.

\section{Bibliografía}

1. Salas-Perea R. Competencia y desempeño profesionales. La Habana: Ciencias Medicas; 1999. p. 51-9.

2. Yurss I. Atención a la familia: otra forma de enfocar los problemas de salud en atención primaria. Instrument Anales 2002 [online]. URL: http://http://www.cfnavarra.es/salud/ anales/textos/vol24/biblio14/bsuple9.html. [08.04.2006].

3. Louro-Bernal I. Matriz de salud del grupo familiar: un recurso para el diagnóstico de la situación de salud de la familia. Rev Cubana Med Gen Integr 2004 [online]. URL: http://www.bvs.sld.cu/revistas/mgi/vol20_3_04/ mgi06304.htm. [19.05.2006].

4. Reingeniería de la residencia en Medicina General Integral. La Habana: Instituto Superior de Ciencias Médicas de La Habana; 2004.

5. Manual de procedimientos básicos del médico y enfermera de la familia. La Habana: MINSAP; 2004.

6. Díaz-Novas J, Gallego-Machado BR. Algunas ideas y reflexiones sobre la atención primaria y el médico de Familia. Rev Cubana Med Gen Integr 2004 [online]. URL: http:// scielo.sld.cu/scielo.php?script=sci_arttext\&pid=S086421252004000500017\&lng=es\&nrm=iso. [23.04.2006]

7. Díaz-Veliz Martínez E, Ramos-Ramírez R. Algunas reflexiones en torno a la atención médica integral a la familia. Rev Cubana Educ Med Super 2002 [online]. URL: http:// www.bvs.sld.cu/revistas/ems/vol16_2_02/VI\%D1etas educaci\%d3n med.jpg. [23.04.2006].

8. Aguilera-García L, Casado-Vicente V. Competencias esenciales en el nuevo programa de medicina familiar y comunitaria. EIDON 2003 [online]. URL: http://www.fcs.es/ fcs/esp/eidon/introesp/eidon12/sumano. [25.04.2006].

9. Toledo-Manrique A, Mazzetti-Soler P, Del Carmen-Sara J, Bustamante-Quiroz R, Salazar-Robles M. Guías de implementación del programa de familias. Lima: Ministerio de Salud; 2005. URL: http://minsa.gob.pe. [27.03.2006].

10. Enrique-Ospina Aguirre J. Atención domiciliaria integral [monografía online]. URL: http://www.monografias.com/ trabajos20/atencion-domiciliaria/atencion-domiciliaria. shtml. [10.05.2006]. 
11. García-Valdés M, Suárez-Marín M. La dimensión asistencial de la ética en la terapia [monografía online]. URL: http://www.psicologia-online.com/colaboradores/mgvaldes/etica.shtml. [30.05.2006].

12. Salas-Mainegra I. Análisis de la calidad de un instrumento para evaluar el nivel de actualización científico técnica de los especialistas de Medicina General Integral. Ciudad de la Habana; 2001

13. Louro BI, Infante PO, De la Cuesta FD. Manual de intervención en salud familiar; 2000. URL: www.infomed.sld. cu.bvs.aps.libros. [29.03.2006]

14. Cerezal-Mezquita J, Fiallo-Rodríguez J. Los métodos científicos en las investigaciones pedagógicas. La Habana: Pueblo y Educación; 2002.

15. Bedoya GA. Evaluación de la calidad de la atención integral a la familia [tesis]. La Habana: Escuela Nacional de Salud Pública; 1998.
16. Cabrera-Pivaral CE, Rodríguez-Pérez I, González-Pérez G, Ocampo-Barrios P, Amaya-López C. Aptitud clínica de los médicos familiares en la identificación de la disfunción familiar en unidades de medicina familiar de Guadalajara. Salud Mental 2006 [online]. URL: http://http://redalyc.uaemex.mx/redalyc/pdf/582/58242906.pdf. [09.01.2007].

17. Chávez-Aguilar V. Aptitud clínica en el manejo de la familia. Rev Med IMSS 2002; 40: 477-81.

18. Díaz E, Medel J, De Pablo R, Palomo L, Toquero F, Pérez F, et al. Criterios básicos para el ejercicio médico en atención primaria [monografía online]. Cáceres: COMECA 2003. URL: http://www.comeca.org/paginas/pdf/atencion_primaria.pdf. [09.11.2006].

19. Muñoz-Sepúlveda. Los lazos de confianza entre el médico y las familias. Rev Med Chile 2004 [online]. URL: http:// www.elmostrador.cl/modulos/noticias/constructor/detalle_noticia.asp?id_noticia=169399. [25.11.2006]. 